\title{
Wieser, Clemens
}

\section{Konzeptualisierungen von Handeln in Paradigmen der Unterrichtsforschung} Zeitschrift für Pädagogik 59 (2013) 1, S. 95-111

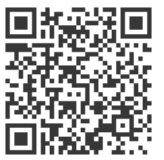

Quellenangabe/ Reference:

Wieser, Clemens: Konzeptualisierungen von Handeln in Paradigmen der Unterrichtsforschung - In: Zeitschrift für Pädagogik 59 (2013) 1, S. 95-111 - URN: urn:nbn:de:0111-pedocs-119286 - DOI: 10.25656/01:11928

https://nbn-resolving.org/urn:nbn:de:0111-pedocs-119286

https://doi.org/10.25656/01:11928

in Kooperation mit / in cooperation with:
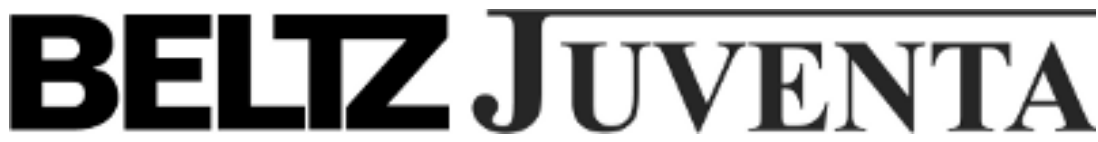

http://www.juventa.de

\section{Nutzungsbedingungen}

Gewährt wird ein nicht exklusives, nicht übertragbares, persönliches und beschränktes Recht auf Nutzung dieses Dokuments. Dieses Dokument ist ausschließlich für den persönlichen, nicht-kommerziellen Gebrauch bestimmt. Die Nutzung stellt keine Übertragung des Eigentumsrechts an diesem Dokument dar und gilt vorbehaltlich der folgenden Einschränkungen: Auf sämtlichen Kopien dieses Dokuments müssen alle Urheberrechtshinweise und sonstigen Hinweise auf gesetzlichen Schutz beibehalten werden. Sie dürfen dieses Dokument nicht in irgendeiner Weise abändern, noch dürfen Sie dieses Dokument für öffentliche oder kommerzielle Zwecke vervielfältigen, öffentlich ausstellen, aufführen, vertreiben oder anderweitig nutzen.

Mit der Verwendung dieses Dokuments erkennen Sie die Nutzungsbedingungen an.

\section{Terms of use}

We grant a non-exclusive, non-transferable, individual and limited right to using this document.

This document is solely intended for your personal, non-commercial use. Use of this document does not include any transfer of property rights and it is conditional to the following limitations: All of the copies of this documents must retain all copyright information and other information regarding legal protection. You are not allowed to alter this document in any way, to copy it for public or commercial purposes, to exhibit the document in public, to perform, distribute or otherwise use the document in public.

By using this particular document, you accept the above-stated conditions of use.

\section{Kontakt / Contact:}

\section{peDOcs}

DIPF | Leibniz-Institut für Bildungsforschung und Bildungsinformation Informationszentrum (IZ) Bildung

E-Mail: pedocs@dipf.de

Internet: www.pedocs.de

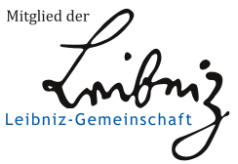



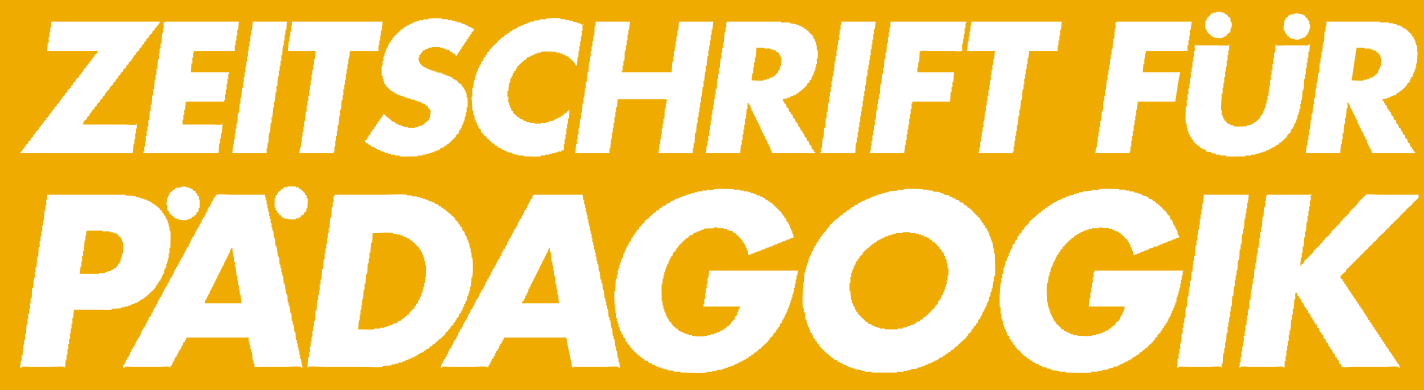

\section{Bildungswissenschaften in der Lehrerbildung}

allgemeiner Teil

Konzeptualisierungen von Handeln in Paradigmen der Unterrichtsforschung

Wer hat, dem wird gegeben? Hochbegabtenförderung und Gerechtigkeit

Selbstthematisierungen von Lehrerinnen und Lehrern zwischen Überhöhung, Normalitätsansprüchen und Verachtung 


\section{Zeitschrift für Pädagogik}

Begründet durch:

Fritz Blättner, Otto Friedrich Bollnow, Josef Dolch, Wilhelm Flitner, Erich Weniger

Fortgeführt von:

Dietrich Benner, Herwig Blankertz, Hans Bohnenkamp, Wolfgang Brezinka, Josef Derbolav, Andreas Flitner, Carl-Ludwig Furck, Georg Geissler,

Oskar Hammelsbeck, Ulrich Herrmann, Diether Hopf, Walter Hornstein,

Wolfgang Klafki, August Klein, Doris Knab, Andreas Krapp, Martinus J. Langeveld, Achim Leschinsky, Ernst Lichtenstein, Peter-Martin Roeder, Wolfgang Scheibe, Hans Scheuerl, Hans Schiefele, Franz Vilsmeier

Herausgeber:

Cristina Allemann-Ghionda (Köln), Sabine Andresen (Frankfurt), Marcelo Alberto Caruso (Berlin), Kai S. Cortina (Michigan), Reinhard Fatke (Zürich), Werner Helsper (Halle), Eckhard Klieme (Frankfurt), Roland Merten (Jena), Jürgen Oelkers (Zürich), Sabine Reh (Berlin), Roland Reichenbach (Basel), Tina Seidel (München), Petra Stanat (Berlin), Heinz-Elmar Tenorth (Berlin), Ewald Terhart (Münster), Rudolf Tippelt (München)

Die Zeitschrift für Pädagogik wird in folgenden Datenbanken und bibliografischen

Diensten ausgewertet:

- CIJE (Central Index to Journals in Education, Phoenix, USA)

- FIS Bildung (Fachinformationssystem Bildung, Frankfurt a.M.)

- PSYINDEX (Zentralstelle für Psychologische Information und Dokumentation, Trier)

- SSCI (Social Sciences Citation Index, Institute for Scientific Information, Philadelphia, USA)

- SOLIS (Informationszentrum Sozialwissenschaften, Bonn)

Geschäftsführende Herausgeber:

Roland Reichenbach (verantwortlich), Kai S. Cortina (Besprechungen), Ewald Terhart, Rudolf Tippelt

Zusammenstellung des Thementeils „Bildungswissenschaften in der Lehrerbildung“: Ewald Terhart 


\section{ZEITSCHRIFT FÜR PADAGOGIK Jahrgan 59- - Heft 1}

\section{Inhaltsverzeichnis}

Thementeil: Bildungswissenschaften in der Lehrerbildung

Olga Kunina-Habenicht/Franziska Schulze-Stocker/Mareike Kunter/

Jürgen Baumert/Detlev Leutner/Doris Förster/Hendrik Lohse-Bossenz/

Ewald Terhart

Die Bedeutung der Lerngelegenheiten im Lehramtsstudium und deren

individuelle Nutzung für den Aufbau des bildungswissenschaftlichen Wissens $\quad \ldots \quad 1$

Lena Rösler/Friederike Zimmermann/Johannes Bauer/Jens Möller/Olaf Köller

Interessieren sich Lehramtsstudierende für bildungswissenschaftliche

Studieninhalte? Eine Längsschnittstudie vom ersten bis zum vierten Semester $\quad \ldots \quad 24$

\section{Johannes König/Martin Rothland}

Pädagogisches Wissen und berufsspezifische Motivation am Anfang der Lehrerausbildung. Zum Verhältnis von kognitiven und nicht-kognitiven Eingangsmerkmalen von Lehramtsstudierenden

\section{Colin Cramer}

Beurteilung des bildungswissenschaftlichen Studiums durch Lehramtsstudierende in der ersten Ausbildungsphase im Längsschnitt

Deutscher Bildungsserver

Linktipps zum Thema „Bildungswissenschaften in der Lehrerbildung“

\section{Allgemeiner Teil}

\section{Clemens Wieser}

Konzeptualisierungen von Handeln in Paradigmen der Unterrichtsforschung

Kirsten Meyer/Benjamin Streim

Wer hat, dem wird gegeben? Hochbegabtenförderung und Gerechtigkeit 
Angelika Paseka

Selbstthematisierungen von Lehrerinnen und Lehrern - zwischen Überhöhung,

Normalitätsansprüchen und Verachtung

\section{Besprechungen}

\section{Christian Brüggemann}

Iulius Rostas (Hrsg.): Ten Years After. A History of Roma School Desegregation in Central and Eastern Europe

Michael Geiss

Michelle M. Nickerson: Mothers of Conservatism: Women and the postwar right

Lars Hoffmann/Poldi Kuhl

Franz Schott/Shahram Azizi Ghanbari (Hrsg.): Bildungsstandards, Kompetenzdiagnostik und kompetenzorientierter Unterricht zur Qualitätssicherung des Bildungswesens. Eine problemorientierte Einführung in die theoretischen Grundlagen

\section{Dokumentation}

Pädagogische Neuerscheinungen

Impressum 


\section{Table of Contents}

\section{Topic: Educational Sciences in Teacher Training}

Olga Kunina-Habenicht/Franziska Schulze-Stocker/Mareike Kunter/

Jürgen Baumert/Detlev Leutner/Doris Förster/Hendrik Lohse-Bossenz/

Ewald Terhart

The Significance of Learning Opportunities in Teacher Training Courses and Their Individual Use for the Development of Educational-Scientific Knowledge

Lena Rösler/Friederike Zimmermann/Johannes Bauer/Jens Möller/Olaf Köller Are Students in Teacher Training Interested in Educational-Scientific Contents?

A longitudinal study covering the first four semesters

\section{Johannes König/Martin Rothland}

Pedagogical Knowledge and Job-Specific Motivation at the Beginning of Teacher Training. On the relation between cognitive and non-cognitive enrollment characteristics among students in teacher training

\section{Colin Cramer}

The Assessment of Educational Scientific Study Courses as Given by Students in Their First Phase of Teacher Training in Longitudinal Section

Deutscher Bildungsserver

Tips of links relating to the topic of "Educational Sciences in Teacher Training"

\section{Contributions}

\section{Clemens Wieser}

Conceptualizations of Action in Paradigms of Research on Teaching

Kirsten Meyer/Benjamin Streim

He Who Has Will Be Given? Promotion of highly gifted students and fairness $\quad . .112$

Angelika Paseka

Self-Conceptualizations of Teachers - Between super-elevation, claims to normality, and contempt

Book Reviews

New Books 


\title{
Allgemeiner Teil
}

\section{Konzeptualisierungen von Handeln in Paradigmen der Unterrichtsforschung}

\begin{abstract}
Zusammenfassung: Konzepte für Unterricht - gefasst als Modelle, Theorien oder Schemata - wurden sowohl in Allgemeiner Didaktik als auch in der Lehr-Lern-Forschung entwickelt. Durch eine Analyse von Konzepten beider Paradigmen sollen Gemeinsamkeiten und Unterschiede in den Konzeptualisierungen von Handeln identifiziert werden. Die Analyse geschieht durch eine Aufschlüsselung von Theorien beider Paradigmen in ihre Komponenten. Ausgehend von identifizierten Herausforderungen der Konzeptualisierung von Handeln werden im Resümee konzeptuelle, methodologische und empirische Perspektiven skizziert, durch die Handeln im Unterricht untersucht werden kann.
\end{abstract}

Schlagworte: Didaktik, Lehr-Lern-Forschung, Handeln, Konzeptualisierung, Unterrichtsforschung

\section{Ausgangslage}

Zumal Konzepte für Unterricht sowohl von Allgemeiner Didaktik als auch Lehr-LernForschung entwickelt werden, kann wissenschaftstheoretisch von der Existenz zweier Paradigmen gesprochen werden, die derzeit kaum aufeinander Bezug nehmen: „Obwohl die Allgemeine Didaktik und die empirische Lehr-Lern-Forschung sich auf den gleichen Gegenstandsbereich beziehen, dominiert zwischen diesen beiden [...] Fremdheit und organisiertes Nicht-zur-Kenntnis nehmen“ (Terhart, 2002, S. 77). Diese Ausgangslage soll zum Einstieg knapp umrissen und kommentiert werden.

\subsection{Präskriptive Zugriffe auf Unterricht durch Allgemeine Didaktik}

Allgemeine Didaktik versucht, für Lehrer einen konzeptuellen Rahmen der Gestaltung von Unterricht anzubieten. An diesen Angeboten wird kritisiert, dass sie in ,seit Jahrzehnten immer gleich strukturierten und erfolgreichen Lehrbüchern“ (Terhart, 2005, S. 12) tradiert und „seit langem festgefahren“ (von Olberg, 2004, S. 119) wären. Eine Ursache hierfür wird darin gesehen, dass Didaktiken in der Regel nicht auf Basis empirischer Forschung entwickelt wurden. Didaktische Konzepte ,sind nicht aus empiri- 
scher Forschung entstanden, sondern weitgehend im Kontext von Lehrerbildung, d.h. auf der Basis von Praktikerwissen“ (Terhart 2002, 81). Spätestens seit durch Studien belegt ist, „dass die im Lehrerstudium [...] erlernten allgemein-didaktischen und fach-didaktischen Theorien [...] im Laufe der Berufsausübung nahezu vollständig verschwinden“ (Wahl, 2001, S. 158), kann von „praktischer“ didaktischer Theorie nicht mehr problemlos gesprochen werden. Das Problem der „unpraktischen“ Didaktik zeigt sich ebenso bei didaktischen Konzepten, die nicht aus „Praktikerwissen“, sondern vor bildungsphilosophischen, schultheoretischen oder kommunikationstheoretischen Hintergründen entwickelt wurden. Trotz dieser vielfältigen Hintergründe zeigt sich eine problematische Gemeinsamkeit: Was im Unterricht zu tun ist, wurde präskriptiv beschrieben. Das dominierende Vorgehen brachte mit sich, dass Didaktik selten ausgehend von empirischer Forschung entwickelt wurde. Dabei besteht in der Didaktik keine generelle Empiriefeindlichkeit: Die von Kaiser, Koch-Priewe und Stübig (2007, S. 15-16) dokumentierten didaktischen Forschungsprojekte machen jedoch deutlich, dass empirische didaktische Forschung insbesondere an der Prüfung und Weiterentwicklung bereits entwickelter Konzepte interessiert war.

Das Fehlen einer direkt aus empirischer Forschung entwickelten didaktischen Theorie, die explizit auf Handeln von Lernenden und Lehrenden Bezug nimmt, ist bis heute ein Problem der Didaktik geblieben: „Fragen der Erforschung, gar noch der empirischen Erforschung, von Unterricht werden [...] nicht genannt" (Terhart, 2002, S. 80). Entsprechend wird in der Didaktik moniert, dass ,ungestillte pädagogische Orientierungsbedürfnisse“ einer „lernerzentrierten Methodenkultur“ den Weg ebnen, in der Lehrer nur noch Meinungen moderieren müssten (von Olberg, 2004, S. 130). Die Kritik richtet sich ebenso gegen Positionen, „die Vermittlung von überhaupt etwas abschaffen, indem sie sich als Kommunikationstrainings, psychotechnische Einstellung und Sinnenstimulation [...] von Unterricht inszenieren" (Gruschka, 2002, S. 11). Spätestens in der selbstkritischen Auseinandersetzung nach dem PISA-Schock wurde allerdings klar, dass ,nur wenige Vertreterinnen und Vertreter der Allgemeinen Didaktik die Wirkungen des alltäglichen Unterrichts [...] mit empirischen Verfahren untersucht haben“" (Meyer, Prenzel \& Hellekamps, 2008, S. 7).

\subsection{Experimentelle Auseinandersetzung mit Unterricht durch Lehr-Lern-Forschung}

Im Gegensatz zur Didaktik wurde in der Lehr-Lern-Forschung üblicherweise nicht der Anspruch erhoben, präskriptiv Leitlinien für das Handeln von Lehrern vorzugeben. In der Lehr-Lern-Forschung wird Lernen als Prozess charakterisiert, bei dem Verlauf und Ergebnis (Produkt) nur bedingt zu steuern sind. Insofern sind Leistungstests, Untersuchungen zu Lernzeit und Lernerfolg oder der Fokus auf kognitive Prozesse des Lernens typisch für die Lehr-Lern-Forschung (Blömeke \& Müller, 2008, S. 240). Charakteristisch ist die Verwendung des Begriffs Lehr-Lern-Forschung in Zusammenhang mit empirischer Forschung und in Distanzierung zu Didaktik; Überschriften wie „Unterrichts- 
forschung als empirisches Komplement der Didaktik“ (Klieme, 2006, S. 765) legen davon Zeugnis ab.

Lehr-Lern-Forschung und Allgemeine Didaktik teilen das Problem, dass sie nur mittelbar auf das Handeln von Lehrern Bezug nehmen. Diese Mittelbarkeit wird - ebenso wie in der Didaktik - auch von Vertretern der Lehr-Lern-Forschung problematisiert. Ein aktuelles Beispiel bietet die Cognitive Load Theory, deren Vertreter selbst bezweifeln, dass experimentell im Labor geprüfte Regeln externe Validität aufweisen und in authentischen Lernsituationen im Unterricht gültig sind (de Jong, 2010, S. 123-125). Ebenso skeptisch argumentiert Gruschka (2002, S. 116) aus didaktischer Perspektive, dass Versuche der Klassifikation von Lernarten oder Lernschritten zwangsläufig in Distanz zu alläglichem Handeln im Unterricht bleiben. Spezifisch für die Lehr-Lern-Forschung ist das Problem, dass ,,im Zuge der weiteren Spezialisierung und Verfeinerung von Fragestellungen und Methoden die Lehr-Lern-Forschung immer mikroskopischer wird wohingegen der Blick der handelnden Lehrer auf ihr Feld natürlich großräumiger und ganzheitlicher ist" (Terhart, 2002, S. 81). Eine andere Erklärung für das Scheitern der klassischen Lehr-Lern-Forschung sieht Staub (2006, S. 172) darin, „dass die Ziele von Bildungsprozessen grundsätzlich immer streitbar bleiben und als kulturelle und politische Fragen nicht allein mittels wissenschaftlichen Methoden geklärt werden können“.

\section{Konzeptualisierungen von Handeln in Theorien des Unterrichts}

In seiner Analyse von Konzepten der Didaktik problematisiert Terhart (1999, 2008) seit langem, dass dort selbst gesetzte konzeptuelle Ausgangspunkte verlassen werden, wenn diese in Handeln im Unterricht übersetzt werden. Bereits früh stellt Terhart (1999, S. 645) die Frage, ob dies beim Übergang von Theorie zu Praxis ein systematisch notwendiges Vorgehen ist oder ob es sich hierbei schlichtweg um einen Fehler in der Theorieentwicklung handelt. Ein Entwicklungsfehler didaktischer Theorien liegt traditionell in der Vorgabe von Zielbestimmungen für das Lehrerhandeln, die nicht ausgehend von empirischer Forschung entwickelt werden. Parallel dazu besteht ein Entwicklungsfehler von Lehr-Lern-Theorien darin, im Unterricht wirkende Faktoren auf Lernen zu benennen, die jedoch ,gegenüber der realen Unterrichtsinteraktion und den konkreten Verläufen sehr abstrakt bleiben“ (Breidenstein, 2010, S. 871).

Beide Fehler in der Theorieentwicklung werden erklärbar, wenn die Beziehung von Theorie und Praxis als Beziehung zwischen Wissen und Handeln verstanden wird. Neuweg (2011, S. 452-454) benennt Handeln als eine dritte Dimension von Wissen, die neben Ausbildungswissen (,Wissen im objektiven Sinne“) und mentalen Strukturen (,Wissen im subjektiven Sinne“) existiert. Unterrichten als spezifische Form des Handelns in Schule ist Neuweg folgend als adaptives Lehrer-Verhalten konzeptualisierbar, das situativ und kontextuell entwickelt wird. Handeln ist gleichzeitig eine Form von Wissen, das für Handelnde in der Regel implizit verfügbar ist und daher nur bedingt verbalisiert werden kann. Über dieses Verständnis von Handeln besteht paradigmenübergreifend kontinuierlich große Einigkeit (Luhmann \& Schorr, 1982; Helsper, 2002; 
Baumert \& Kunter, 2006). Deutlich weniger Konsens besteht darüber, wie Handeln im Unterricht valide zu erfassen und zu analysieren ist.

Für die Analyse von Konzeptualisierungen von Handeln im Unterricht ist es sinnvoll, Theorien der Didaktik und der Lehr-Lern-Forschung aufgeschlüsselt in Komponenten zu untersuchen. Herausgearbeitet wird, wie Handeln in Konzepten des Unterrichts festgemacht wurde. Die Aufschlüsselung der Theorien in Komponenten folgt methodisch der Vorstellung von Schurz (1993, S. 9), der Theorien als ,,mehrkomponentige kognitive Systeme“" versteht. Solche Systeme enthalten nach Schurz folgende Komponenten:

(1) Der Theoriekern besteht aus charakteristischen Annahmen über das Erkenntnisobjekt sowie aus Modellvorstellungen, die Mechanismen und Zusammenhänge beschreiben. Annahmen und Modellvorstellungen werden als Axiome aufgestellt und bilden den ontologischen Rahmen der Forschung.

(2) Die methodologische Komponente enthält einerseits methodische Regeln, wie das Forschungsobjekt zu untersuchen ist; andererseits enthält sie epistemologische Annahmen, etwa die Bestimmung des Verhältnisses von Forschungsobjekt und Forschungssubjekt und Annahmen zu Interesse und Fokus der Forschungsarbeit.

(3) In der programmatischen Komponente wird das - in der Regel weitgehend uneingelöste - Versprechen formuliert, die Phänomene des zu untersuchenden Zusammenhangs zu erklären. Die Programmatik wird generalisierend aus exemplarischen Forschungsarbeiten entwickelt.

Das von Schurz entwickelte analytische Schema wird in Folge genutzt, um Komponenten von Theorien der Didaktik und der Lehr-Lern-Forschung zu vergleichen. Im Vergleich sollen Gemeinsamkeiten und Bruchstellen zwischen den Theorien beider Paradigmen systematisch aufgedeckt werden. In den folgenden Abschnitten werden (2.1) ontologische Annahmen und Modellvorstellungen der Theoriekerne, (2.2) methodologische Komponenten und (2.3) programmatische Komponenten von Theorien der Didaktik und der Lehr-Lern-Forschung verglichen. Auf diese Weise werden einige konzeptuelle, methodologische und empirische Herausforderungen in der Entwicklung von Theorien des Unterrichts sichtbar gemacht.

\subsection{Konträre ontologische Annahmen und Modellvorstellungen im Theoriekern}

Wesentliche Unterschiede finden sich in der Konzeption von Handelnden in Didaktiken und Lehr-Lern-Forschung. In der Didaktik werden Handelnde traditionell als autonome Subjekte beschrieben. Das Subjekt ist hier ein sich selbst gewisses und bestimmendes Ich-Bewusstsein, das dem Nicht-Ich, der das Subjekt umgebenden Welt, begegnet. In der Lehr-Lern-Forschung hingegen werden Handelnde als Individuen konzipiert, die nach einer Aufrechterhaltung von psychosozialer Funktionstüchtigkeit streben (Herber, 
1997, S. 42-46). Die Unterschiede in der Konzeption von Handelnden spiegeln sich auch in Modellen von Lehren und Lernen wider. In der Lehr-Lern-Forschung wird Lehren in der Regel nicht als Interaktion, sondern als effiziente Instruktion gefasst. Diese Fassung kann als Grund dafür gesehen werden, warum Erkenntnisse der Lehr-LernForschung kaum Eingang in das Unterrichten von Lehrern gefunden haben. „Fundamental learning and teaching research tends to focus on efficiency, i.e., how much can be learned from a given amount of effort or time. It is proposed here that these aims do not mesh well with the organizational logic of every instructional enterprise" (Rothkopf, 2008, S. 365). Die Organisation von Lehren und Lernen im Unterricht ist entgegen der Lehr-Lern-Forschung nicht auf die Maximierung von Effizienz im Lernprozess ausgerichtet. Darüber hinaus baut Lehr-Lern-Forschung auf Vorstellungen der Kognitionsforschung auf und konzipiert Unterricht in der Regel nicht als Handeln zwischen Lehrenden und Lernenden (Giesinger, 2006; Reusser, 2008). Dem effizient zu instruierenden Individuum der Lehr-Lern-Forschung steht in der Didaktik ein Subjekt gegenüber, das zur mündigen Partizipation an Gesellschaft erzogen werden soll. So wird in der bildungstheoretischen Didaktik vom „Recht auf Selbstsein des Subjekts“ (Blankertz, 1975, S. 45) ausgegangen. In der kritisch-kommunikativen Didaktik wird das Subjektkonzept im Ziel sichtbar, ,,vorhandene Wirklichkeiten kritisch zu reflektieren und sie in anspruchsvollere Möglichkeiten zu transferieren“ (Winkel, 2002, S. 95). Auch die konstruktivistische Didaktik steht nach Reusser (2006, S. 156-157) „dem modernen Selbstideal der Autonomie“ nahe und zielt auf die „Freisetzung von Subjektivität und von Verantwortung für das eigene Wissen“. Die genannten didaktischen Theorien zielen allesamt darauf ab, Subjekte zur Mündigkeit zu befähigen. In der Konzeption der Handelnden bestehen also beträchtliche Unterschiede zwischen den beiden Paradigmen, innerhalb scheinen die Konzeptionen von Subjekt bzw. Individuum jedoch relativ stabil und unumstritten. Allerdings wird anhand der genannten ontologischen Annahmen der Didaktik sowie der Lehr-Lern-Forschung deutlich, dass Handeln konzeptuell nicht in Theorien integriert wurde. Handeln ist somit eine konzeptuelle Leerstelle in Theorien beider Paradigmen.

\subsection{Komplementäre Forschungsinteressen in methodologischen Komponenten}

Trotz konträrer Annahmen in den Theoriekernen werden methodologische Zugänge beider Paradigmen in Forschungsprojekten gemeinsam eingesetzt. Konfrontationen beim Aufeinandertreffen methodologischer Positionen werden beispielhaft von Messner (2007, S. 48-55) anhand eines Forschungsprojekts zum „,selbstständigkeitsorientierten aufgabengesteuerten Unterricht“" illustriert. Das Forschungsprojekt wurde ausgehend von einem didaktischen Konzept (selbstständiges Lernen) und einem lehr-lern-theoretischen Konzept (selbstreguliertes Lernen) entwickelt. Messner (2007, S. 45) beschreibt, dass sich während der Entwicklung des Forschungsdesigns ,eine zunächst aus der didaktischen Tradition hergeleitete Forschungsidee [...] unter dem Einfluss lehr-lerntheo- 
retischer Forschungsparadigmen in neuer Form herausgebildet hat". Innerhalb des Projekts sieht Messner die Chance, Möglichkeiten der kognitiven Steuerung von Lernen auszuweiten, zu der die Didaktik weniger differenzierte Konzepte vorzuweisen hat. Gleichzeitig sieht er die Gefahr, dass bildungsphilosophische Aspekte didaktischer Konzepte wie „Autonomie und Widerständigkeit“" verloren gehen. Dieser Konflikt wurde zu Gunsten einer quasi-experimentellen Untersuchung von selbstreguliertem Lernen aufgelöst. In der Vorstellung des Forschungsprojekts ,zeigt sich, dass die Allgemeine Didaktik und die Lehr-Lern-Forschung im konkreten Fall in starke Spannung zueinander geraten. Diese wird in der empirischen Forschungspraxis meist zugunsten einer der Lehr-Lern-Forschung entsprechenden Konzeptualisierung und methodologischen Operationalisierung aufgelöst. Tatsächlich bedürfte gerade diese empirische Zurichtung einer selbstbewusst-substanziellen Theoretisierung" (Messner, 2007, S. 43). Durch eine solche Theoretisierung erhofft Messner, den oben genannten Gefahren einer einseitigen Konzeptualisierung der Lehr-Lern-Forschung entgegenzuwirken. Die konträren ontologischen Annahmen werden methodologisch einerseits reproduziert, zumal die Operationalisierung zugunsten eines Paradigmas ausfällt. Gleichzeitig wird Didaktik aber komplementaristisch genutzt, um Probleme der Lehr-Lern-Forschung durch eine didaktische Rahmung auszugleichen.

Ein weiteres Beispiel für den komplementären Einsatz von methodologischen Konzepten beider Paradigmen ist die Modellierung von Kompetenzen, die von Klieme und Rakoczy (2008) einerseits als Produkt, andererseits als Prozess des Lernens charakterisiert werden. An das Kompetenz-Konzept wird die Erwartung geknüpft, dass erworbene Kenntnisse und Fähigkeiten nicht als träges Wissen, sondern anwendungsorientiert und transferfähig entstehen. Durch das Einfließen wissensbezogener, handlungsbezogener und subjektbezogener Dimensionen - analog zu den Dimensionen von Wissen bei Neuweg (2011) - wird die empirische Operationalisierung in der Lehr-Lern-Forschung als Herausforderung wahrgenommen. Derart modellierte Kompetenzen sollen einerseits gemessen werden, andererseits sind aber auch Prozessmerkmale des Unterrichts zu erforschen, durch die das gewünschte Niveau des Produkts erreicht wird. Die gewonnenen Kriterienkataloge der Prozessqualität von Unterricht werden insbesondere aus professionalitätstheoretischer Sicht kritisiert, zumal die einzelnen Kriterien ,hohe Suggestivkraft“ entfalten, wobei ,deren Einzelbedeutung schwer abzuschätzen ist“" (Herzog \& Markova, 2011, S. 70). Wie Unterricht zu gestalten ist, um Transfer zu erzielen, wird in der Lehr-Lern-Forschung zwar beforscht und konzeptuell für Unterricht seit geraumer Zeit aufbereitet (etwa Paas, 1992) - allerdings geben die Konzepte primär Vorschläge, wie und welche kognitiven Prozesse im Lernen durch Instruktion bevorzugt aktiviert werden sollen. Entsprechend ergänzen Klieme und Rakoczy (2008) das lehr-lern-theoretische Konzept der Kompetenz um Konzepte der Unterrichtsgestaltung, die sie vor allem in Fachdidaktiken verorten und als Strategien der Verwirklichung von Kompetenz verstehen: „Mittels authentischer, ganzheitlicher, lebensnaher Lernaufgaben will man nicht bloß einzelne Fertigkeiten trainieren oder gar ,träges Wissen 'vermitteln, sondern zur Bewältigung komplexer Anforderungssituationen befähigen“ (Klieme \& Rakoczy, 2008, S. 223). Klieme und Rakoczy nutzen die Fokussierungen beider Paradig- 
men für jeweils unterschiedliche Herausforderungen: Einerseits zielt die Modellierung von Kompetenzen darauf ab, Empfehlungen für eine Schulreform zu geben und der Vermittlung von trägem Wissen entgegenzuwirken; andererseits soll die (fach-)didaktische Entwicklung von Lernaufgaben mit den genannten Eigenschaften dazu führen, Kompetenzen durch Unterricht zu erreichen. Verbleibt jedoch der Modus der Entwicklung von Lernaufgaben in der präskriptiven Tradition, bleibt empirisch unerforscht, wie Lernaufgaben zwischen Lehrern und Schülern in der natürlichen - das heißt nicht experimentell verfremdeten - Situation des Unterrichts verhandelt werden. Diese Leerstelle unterstreicht das methodologische Desiderat, Wege der Untersuchung von Pädagogik als Praxis der Erziehung, Vermittlung und Bildung in der Form ihres Auftretens im Unterricht zu entwickeln (Gruschka, 2011, S. 209).

\subsection{Gleiche Phänomene der Praxis, Nähe der programmatischen Komponenten}

Ebenso wie in methodologischen Komponenten von Theorien zeigt sich in den programmatischen Komponenten zunehmende Anerkennung von Komplementarität zwischen Paradigmen. Im Gegensatz zu den divergenten methodologischen Zugängen deuten mehrere Studien jedoch darauf hin, dass Theorien beider Paradigmen programmatisch das konvergente Ziel verfolgen, Handlungen von Lehrern und Schülern in Konzepten für Unterricht zu fassen. Folglich ist nicht von zwei eigentümlichen, sondern von einer geteilten Programmatik zu sprechen. So stellen Blömeke und Müller (2008, S. 241-243) „Phänomengruppen des Unterrichtsgeschehens“" vor, die sowohl von Allgemeiner Didaktik als auch von Lehr-Lern-Forschung untersucht werden. Sie rechnen hierzu (1) die systematische Analyse von Aufgaben, die im Unterricht gestellt werden, (2) Sozialformen des Unterrichts und daran gekoppelt (3) Akteure des Unterrichts und deren Interaktion im Unterricht sowie (4) die Kommunikationsebene (Inhalt bzw. Form), auf der interagiert wird. Analog dazu benennt Reusser (2008, S. 230) strukturierte Unterrichtsführung, unterstützendes Sozialklima und kognitive Aktivierung als „Basisdimensionen guten Unterrichts“. Er hält fest, dass sich ,zahlreiche Parallelen und Übereinstimmungen“ zwischen Allgemeiner Didaktik und Lehr-Lern-Forschung finden lassen, wenn die beiden Paradigmen verglichen werden. In beiden Fällen werden konsensualisierte Begriffe für Prozesse des Handelns im Unterricht gesucht. Diese Konsensualisierung kann als Versuch verstanden werden, eine gemeinsame transdisziplinäre Sprache zu entwickeln. Transdisziplinäre Sprache ermöglicht eine Homogenisierung von Begriffen gegenüber Phänomenen, wodurch Beschreibungen und Analysen desselben Phänomens vergleichbar werden und triangulierend auf die Konzeptualisierung des komplexen Phänomens wirken. Entsprechend resümieren Blömeke und Müller (2008, S. 253), ,dass sich Merkmale der Allgemeinen Didaktik und der Lehr-Lernforschung möglicherweise gut zu übergreifenden Strukturen des Unterrichtsgeschehens zusammenfassen lassen“. Programmatisch halten sie fest, dass sowohl Lernpsychologie als auch Didaktik sich auf gemeinsame Phänomene der Praxis des Unterrichts beziehen. Auch Reusser (2008, S. 220) spricht program- 
matisch von gemeinsamen Grundfragen, gibt aber zu bedenken, dass nach aktuellem Forschungsstand noch nicht von einer gemeinsamen Auseinandersetzung mit Unterricht zu sprechen ist: „Auch wenn es im Prinzip dieselben Grundfragen nach Lehren und Lernen sind, welche die in der Lehrerbildung verankerte Allgemeine Didaktik und die an Psychologie und Empirie orientierte Lehr-Lernforschung bearbeiten, sehen sich die beiden Bereiche $[. .$.$] immer noch eher als ,fremde Schwestern'." Der Versuch, Phänomengrup-$ pen (Blömeke \& Müller, 2008) bzw. Basisdimensionen (Reusser, 2008) festzumachen, macht deutlich, dass sich die Paradigmen in Bezug auf die Praxis des Unterrichtens mit denselben Phänomenen beschäftigen. Entsprechend ist festzuhalten, dass trotz der Konkurrenz von Allgemeiner Didaktik und Lehr-Lern-Forschung die Paradigmen nicht zwei jeweils eigentümliche, sondern vielmehr eine gemeinsame Programmatik verfolgen. Diese wird jeweils spezifisch in der Sprache eines Paradigmas artikuliert, zielt jedoch dennoch darauf ab, Phänomene des Unterrichts diskutierbar zu machen.

\section{Perspektiven für die Analyse von Handeln im Unterricht}

Benannte Phänomengruppen bzw. Basisdimensionen können als Entwicklung einer analytischen Sprache gelesen werden, die eine systematische Auseinandersetzung mit Unterricht ermöglichen soll. Für Lehrer bietet eine solche analytische Sprache Möglichkeiten, Handlungen im Unterricht zu interpretieren. Solche Interpretationsmöglichkeiten können für Lehrer orientierend und für ihr Handeln entlastend wirken. Ausgehend von bislang identifizierten Herausforderungen sollen abschließend konzeptuelle und methodologische Perspektiven sowie empirische Forschungsprogramme vorgestellt werden, durch die das programmatische Ziel der Entwicklung einer analytischen Sprache über Unterricht angenähert werden kann.

\subsection{Charakteristika von Handeln im Unterricht}

Die Analyse konzeptueller Bestimmungen - das heißt in Paradigmen verankerter ontologischer Annahmen und Modellvorstellungen - hat gezeigt, wie unterschiedlich Schüler - von zur Autonomie zu führenden Subjekten bis zu effizient zu instruierenden Individuen - konzeptualisiert werden. In beiden Varianten ist es jedoch notwendig, das soziale Verhältnis von Schülern und Lehrern näher zu bestimmen. Dieses Verhältnis wird in der Regel als Interaktion gefasst, durch die ,sozialer Sinn konstituiert, reproduziert und modifiziert wird“ (Lüders, 2011, S. 644). Interaktion ist grundsätzlich durch doppelte Kontingenz geprägt, das heißt: Der Sinn der Handlung einer Person wird von einer anderen Person erst durch Interpretation erschlossen. Diese Interpretation ist kontingent, also für die Person plausibel, aber nicht notwendig richtig. Zumal diese Interpretation von beiden Personen geleistet wird, besteht doppelte Kontingenz. Diese Denkfigur macht deutlich, dass Handlungen von Lehrern zu Handlungen von Schülern führen können, die nicht intendiert waren. Eine von Schülern erwartete Handlung kann eintreten, 
muss es aber nicht. Dieser Umstand wurde von Luhmann und Schorr (1982, S. 12) als Technologieproblem der Didaktik bezeichnet: Didaktik kann nicht als Technologie konzipiert werden, weil einer Technologie ein klar definiertes kausales Verhältnis zugrunde liegen muss, in der Beziehungen vollständig beschrieben sind. Eine solche Beschreibbarkeit ist in Interaktionen - das heißt auch in Unterricht - nicht gegeben.

Im Unterricht sind die Ziele des Lehrers, Zweck der Ziele und Mittel, mit denen Ziel und Zweck erreicht werden sollen, der Interpretation durch Schüler ausgeliefert. Erst in Folge ihrer Interpretation handeln Schüler bezogen auf Ziele, Zwecke und Mittel des Lehrers. Ebenso sind Lehrer darauf angewiesen, die ausgedrückten Interpretationen von Schülern im eigenen Handeln zu berücksichtigen, sofern zwischen Schülern und den im Unterricht präsentierten Zielen, Zwecken und Mitteln vermittelt werden soll. Die beiderseits geleisteten Handlungen und Interpretationen prägen den Verlauf von Unterricht. Um der beschriebenen Programmatik nachzukommen, eine analytische Sprache für Phänomengruppen bzw. Basisdimensionen des Unterrichts zu entwickeln, ist es konzeptuell notwendig, doppelte Kontingenz als Eigenschaft von Interaktion ontologisch festzuschreiben und so die Entstehung einer reflexiven Leerstelle zu vermeiden. Die illustrierten handlungstheoretischen Grundlagen machen deutlich, dass Theorien des Unterrichts Wege der systematischen Reflexion über Handlungen im Unterricht zeigen müssen. Diese Notwendigkeit wird auch international in der Debatte um Pädagogik als Komplexitätswissenschaft unterstrichen. Aktuelle pädagogische Theorie bildet die Komplexität von pädagogischem Handeln nur unzureichend ab und bietet gerade deshalb keine Grundlage für die Reflexion von Handeln. So argumentieren Jörg, Davis und Nickmans (2007, S. 148): „Current theories render the complexity of educational reality not only largely unreflective, but even difficult to perceive as sites of reflection." Ana$\log$ zur didaktischen Diskussion wird betont, dass Reflexivität und Theorie ausgehend von komplexer Realität des Unterrichts entwickelt werden müssen. Für zukünftige Theorien ist es notwendig, die Möglichkeiten der Darstellung von Komplexität gegenüber sozialen Phänomenen zu berücksichtigen und Charakteristika von Theorie-Komponenten nicht zu trivialisieren (Gage, 2009, S. 11-21). Die Folge von solchen Trivialisierungen wären Theorien, in denen ein korrelativer Zusammenhang zwischen Lehraktivitäten und Lernergebnissen kausal interpretiert wird, ohne mit komplexen Wechselwirkungen „Zwischen Schülermerkmalen, den Lern- und Unterrichtserfahrungen einer Schulklasse und dem methodischen Lehrhandeln" zu rechnen (Lüders \& Rauin, 2008, S. 738).

Wege des Umgangs mit ebendiesen komplexen Wechselwirkungen werden in der Didaktik entwickelt. Deshalb soll in Folge ein Überblick über Ebenen gegeben werden, in denen Handeln im Unterricht aus didaktischer Sicht untersucht wird.

\subsection{Ebenen des Handelns im Unterricht}

Für Beobachtungen und Interpretationen ist es notwendig, typische Ebenen des Handelns im Unterricht zu beschreiben. Gruschka (2011) benennt Erziehung, Vermittlung und Aneignung sowie Bildung als solche typischen Ebenen. Der professionstheoreti- 
sche Konsens über diese Ebenen wird von Helsper (2011, S. 166) verdeutlicht, der resümierend festhält, dass „Lehrerprofessionalität im Kern um Vermittlung und Aneignung, Person und Sache zentriert" wird. Diese Ebenen spiegeln sich in der Lehr-Lern-Forschung, wo Handeln der Lehrperson in den Ebenen Erziehung („Organisation unterrichtlicher Aktivitäten“, „Evaluation“) und Vermittlung und Aneignung (,Zielorientierung“, „Ausführung von kognitiv anspruchsvollen Lernaktivitäten“, „Lernbegleitung“) untersucht wird (Seidel, 2011, S. 611). In diesen Ebenen spiegelt sich ein kritisches Unterscheidungsmerkmal zwischen Didaktik und Lehr-Lern-Forschung: Didaktik ist ,in enger Bezugnahme auf Ideen der Aufklärung entstanden und enthält als konstitutives Element die Kategorie der Bildung“, wohingegen Lehr-Lern-Forschung diese Kategorie nicht semantisch lädt und damit auch nicht als Ebene des Handelns untersucht (Arnold, 2009, S. 33). Zentrale Annahmen zu den drei genannten Ebenen des Handelns im Unterricht sollen in Folge charakterisiert werden.

Bildung wird insbesondere in der Didaktik als Ziel benannt, das in und durch Unterricht erreicht werden soll. Gleichzeitig zeigt sich in empirischen Analysen eine überwältigende Übereinstimmung darin, ,wie in eingeschliffenen Kommunikationsmustern des Unterrichts der bildende Gehalt der Sache und die Vermittlung zwischen Person und Sache ins Leere läuft“ (Helsper, 2011, S. 159). Nachvollziehbar wird Bildung als Handeln im Unterricht nur in ihren Ausgangspunkten, den ,bildenden Potentialen“ (Helsper, 2011, S. 161) des Unterrichts und deren Annahme durch Schüler. Zumal bildende Potentiale des Unterrichts Bildungshandlungen nur ermöglichen und diese nicht auf Unterricht beschränkt sind, sind Phänomene der Bildung dort nur sehr eingeschränkt beobachtbar. Diese Einschränkung wird insbesondere in jenen Forschungsrichtungen deutlich, die sich explizit mit Bildung in und durch Schule beschäftigen: Alheit und Dausien (2009, S. 715) verstehen Bildung aus Sicht der Biographieforschung nicht als ,einzelne Schritte der Aneignung“, sondern als „Prozesse der Verarbeitung, Verknüpfung und (Trans-)Formation von Lernprozessen“, die in ihrer ,biografischen Erfahrungsgestalt" und damit nicht im Unterricht nachvollziehbar werden. Analog dazu wird auch in der Bildungsgangforschung Bildung als ein „Prozess der Transformation oder bewussten Aufrechterhaltung des Welt- und Selbstverhältnisses eines Individuums“" verstanden (Hericks, 2008, S. 64).

Die Ebene der Erziehung ist durch negative und positive Momente gekennzeichnet. Negativ findet Erziehung, ,in ihrer herkömmlichen Form als Gegeneinwirkung statt" (Gruschka, 2011, S. 221), weshalb Lehrer als Erzieher immer mit Widerspruch gegen ihre moralische Kritik konfrontiert sind; Erziehungshandlungen sind jene Handlungen von Lehrern, durch die das Handeln von Schülern ,,unter die moralische Kritik durch den Erwachsenen“ gelangt. Analog wird in der Lehr-Lern-Forschung „effiziente Klassenführung als notwendige Voraussetzung jedes anspruchsvollen Unterrichts“ genannt (Helmke, 2011, S. 637). Positiv gefasst ist Erziehen an das bildungsphilosophische Ziel gekoppelt, Schüler zur Entwicklung eines ,erkenntnisfördernden Habitus“ anzuleiten (Gruschka, 2009, S. 11). Ebenso wird in der Lehr-Lern-Forschung die Schaffung eines lernförderlichen Klimas als zentrales Merkmal der Lernwirksamkeit von Lehrerhandeln genannt (Helmke, 2011, S. 639). Handlungen auf der Ebene 
des Erziehens sind im Unterricht notwendig, wenn die Vermittlung zwischen Schülern und Sache - das „Primat der Wissensvermittlung“ (Oevermann, 1996, S. 144) gestört ist und die gemeinsame Arbeit an einem Gegenstand daher nicht stattfinden kann. Versuche, die Wirkung von Erziehung auf Lernen (bzw. Aneignung) zu erklären, stehen dabei vor dem Problem, dass ein einfacher Zusammenhang nicht hergestellt werden kann: ,Vielmehr deuten die Ergebnisse darauf hin, dass die Qualitäten der Umsetzung verschiedener Organisationsformen für Lernen entscheidend sind“" (Seidel, 2011, S. 615).

Auf Ebene der Vermittlung und Aneignung handeln Lehrer als Vermittler zwischen Schülern und Sache. Vermittlung und Aneignung haben daher sowohl eine sachliche als auch eine lebensweltliche Seite: Die Sache tritt im Unterricht in der Regel in Form von Gegenständen auf, die diese repräsentieren. Die Sache ist in Gegenständen nicht einfacher (das heißt didaktisch reduzierter) darzustellen, als sie ist, sondern ,so einfach zu machen wie möglich, ohne das zu verlieren, was als sachlicher Grund für die Vermittlung angegeben wurde" (Gruschka, 2011, S. 244). Durch diese Position wird ein zumindest minimaler Realismus (Schurz, 2006, S. 26) gefordert, der die Vermittlung auf sachlicher Seite nicht zu einer gänzlich subjektiven Verhandlung von Meinungen macht. Gegenüber der Sache handeln Schüler in der ,lebensweltlichen Pragmatik der erkenntnisleitenden Interessen“ (Gruschka, 2009, S. 43). Diese Pragmatik - und nicht etwa die sachliche Expertise von Lehrern - ist notwendigerweise Ausgangspunkt für Vermittlung (Gruschka, 2009, S. 479). Analog zu diesem Verständnis von Vermittlung konzipiert Gage (2009, S. 61) vor paradigmatisch anderem Hintergrund Lehren: „Teaching encompasses what teachers do in helping their students learn and perform the tasks." Aneignungen entwickeln sich, indem Schüler sich mit Gegenständen auseinandersetzen, Wissen über das Repräsentierte ausgehend von diesen Gegenständen aufbauen und damit Verfügung über die Sache erhalten. Anhand der Gegenstände im Unterricht werden Begriffe, Kontext und Konzepte verhandelt und die Sache beschrieben, erklärt oder beurteilt. Eine Differenz zu Konzeptualisierungen von Vermittlung und Aneignung in der Lehr-Lern-Forschung ist die Annahme, dass die Ziele der Aneignung nicht von Schülern gesetzt werden, sondern von Lehrern. „Diese Ziele müssen [von Schülern] internalisiert und als eigene Lernziele angenommen werden", denn Studien belegen für diesen Fall ,positive Wirkungen auf das konzeptuelle Verständnis und die Wissensentwicklung der Lernenden“" (Seidel, 2011, S. 615-616). In dieser Annahme zeigt sich ein Unterschied in der Konzeption von Handelnden: Die Annahme der Lehr-Lern-Forschung, dass Aneignung ausgehend von Lehrer-Zielen stattfinden soll, ist unvereinbar mit der didaktischen Annahme, dass Schüler sich Wissen aneignen, indem sie ihren erkenntnisleitenden Interessen folgen. Dieser ontologische Unterschied wird auch in Überlegungen von Blömeke (2009, S. 19) zur Analyse von Aufgaben im Unterricht deutlich. Die didaktische Analyse von Aufgaben würde ,ganz anders aussehen, als es derzeit in der Lernforschung der Fall ist“, weil neben der „Bestimmung des objektiven Potentials einer Aufgabe“ didaktisch untersucht wird, wie dieses Potential von Handelnden ausgeschöpft wird. Aus didaktischer Sicht interessant sind Aufgaben in der Form, wie sie im Unterricht behandelt werden, das heißt ,,als vielfach editierte natürliche Protokolle“ 
(Gruschka, 2011, S. 248). In beiden Beispielen wird deutlich, dass trotz unterschiedlicher ontologischer Annahmen sowohl Didaktik als auch Lehr-Lern-Forschung Handeln auf Ebene von Vermittlung und Aneignung thematisieren.

\subsection{Methodologische Wege der Untersuchung von Handeln im Unterricht}

Nachdem die Ebenen von Handeln im Unterricht konzeptuell in mehreren Ebenen beschrieben wurden, sind in Folge methodologische Wege der Analyse von Handeln und Interaktionen zu bestimmen. Ein Charakteristikum solcher Wege ist, dass sie ein Reflektieren von Handeln im Unterricht ermöglichen sollen. Die geringen Möglichkeiten der Reflexion von Handeln im Unterricht spiegeln sich methodologisch in der experimentellen Ausrichtung empirischer Forschung und der Tendenz zur Spezialisierung der Fragestellungen. In der Diskussion der methodologischen Komponenten der Theorien wurde Messner (2007) folgend problematisiert, dass Phänomene des Unterrichts methodologisch verformt werden. Dieses methodologische Problem wird von Terhart (2002, S. 84) auf eine spezifische Blickperspektive auf Unterricht zurückgeführt: „Didaktiker beschränkten sich selbst in ihrer Blickperspektive [...] auf die Mikroprozesse des Lehrens und Lernens. Einen analogen Vorwurf kann man zu Recht der empirischen LehrLern-Forschung machen." Gruschka (2002, S. 88) sieht für dieses Problem zwei methodologische Ursachen: Erstens wird eine „fiktive Praxis“ konstruiert, durch die Handeln im Unterricht nicht erreicht wird. Zweitens beschränkt sich empirische didaktische Forschung auf das Testen und Weiterentwickeln bereits formulierter Konzepte. Alternativ schlägt Gruschka vor, didaktische Forschung zu betreiben, die Unterricht in der Totalität seines Auftretens untersucht. Zu einem ähnlichen Urteil kommt Reusser (2008, S. 224), der prospektiv festhält: „Die empirische Bildungsforschung täte gut daran, sich [...] proaktiv mit der Phänomenologie schulisch-unterrichtlichen Handelns [...] vertraut zu machen." Didaktische Theorien des Unterrichts sind methodologisch zu modernisieren, indem sie sich, „ohne die eigenen Fragestellungen aufzugeben, konsequent an die empirische Bildungs- und Unterrichtsforschung annähern und dadurch zu einem tieferen Verständnis der [...] zu kultivierenden Prozessqualitäten des Lehrens und Lernens" beitragen (Reusser, 2008, S. 232).

Ist es das Ziel, Lehrern durch Theorie eine Möglichkeit der Reflexion von alltäglichem Unterricht und darin ablaufenden Handlungen anzubieten, ist es sinnvoll, konzeptuell fassbar zu machen, wie mit Kontingenz im Unterricht situativ umgegangen werden kann. Zumal das Handeln von Lehrern im Unterricht immer situativ auf ebenso kontingentes Handeln von Schülern bezogen ist, kann auch eine handlungstheoretisch entwickelte Theorie keine teleologische Anleitung für Unterrichten bieten. Entsprechend fungiert handlungstheoretisch entwickelte Theorie als Reflexionshintergrund für Handeln im Unterricht. Der Unterschied zu präskriptiven Theorien liegt darin, dass explizit Wege der Reflexion von Ereignissen im Unterricht gegeben werden. Gegenüber anderen Theorien des Unterrichts hat handlungstheoretisch entwickelte Theorie den Vorteil, Interaktion im Unterricht realistischer zu konzeptualisieren. Gruschka (2002, S. 15) bezeichnet 
solche Ansätze als Theorien und Modelle des Unterrichts und unterscheidet diese von „Theorien und Modelle[n] für Didaktik“. Aufgrund realistischer handlungstheoretischer Konzeptionen entsteht eine Möglichkeit der Reflexion, denn durch sie können systematische Interpretationen von Handlungen im Unterricht entwickelt werden. Reflexivität kann aber auch ohne ausformulierte Theorie entwickelt werden, etwa durch ein Üben im Interpretieren von Unterricht, wie es in qualitativen Forschungsgruppen praktiziert wird (Dausien, 2007, S. 7). In beiden Fällen können Lehrer Strategien der systematischen Interpretation von Handlungen nutzen, um Routinen der Interpretation von kontingenten Handlungen zu entwickeln. Diese Routinen können im Unterricht heuristisch eingesetzt werden, um die Interpretation kontingenter Handlungen von Schülern zu erleichtern.

\subsection{Empirische Forschung zu Handeln im Unterricht}

Entwickelt werden Heurisiken durch ein Üben im Interpretieren von Handlungssequenzen im Unterricht. Das Üben im Interpretieren von Unterricht kann unterstützt werden, indem empirisch fundierte Konzeptualisierungen von Erziehung, Vermittlung und Aneignung oder Bildung zur Verfügung gestellt werden, die für Phänomene des Unterrichts sensibilisieren. In Folge sollen exemplarisch zwei Forschungsprogramme skizziert werden, die diese Unterstützung leisten und programmatisch Wege zu einer empirisch fundierten Theorie des Unterrichts zeigen. Beide entwickeln eine analytische Sprache über Handeln im Unterricht und stellen Strategien zur systematischen Reflexion von Unterricht bereit.

Das Forschungsprogramm von Gruschka zielt darauf ab, durch die Analyse der ,,im Unterricht thematisierten Erziehungsaufgaben und der aufbrechenden Bildungsepisoden“ (Gruschka, 2005, S. 42) eine Theorie des Unterrichts zu entwickeln. Die pädagogische Erfahrung von Unterricht durch Lehrer soll wissenschaftlich erschlossen werden, um das implizite Handlungswissen von Lehrern und die damit verbundene ,nicht zu überbietende kasuistische Erfahrung mit der Vermittlung“" (Gruschka, 2011, S. 246) in intersubjektives Wissen zu überführen. Ausgehend von Transkripten von Unterrichtsstunden rekonstruiert Gruschka objektiv-hermeneutisch das pädagogische Geschehen im Unterricht. Rekonstruiert werden sollen Übergänge im Handeln zwischen Vermittlung, Erziehung und Bildung, wobei einzelne Stunden nicht selten Widersprüche zwischen einer ,pädagogisch unbefriedigenden Kommunikation [...] und der relativen Reibungslosigkeit des kooperativen Vollzugs“ zeigen (Gruschka, 2010, S. 71). Gewonnen werden soll eine allgemeine Strukturtheorie des Unterrichts, die Lehrer für die Entwicklung von Heuristiken nutzen können.

Ähnlich verfolgt Breidenstein (2010, S. 882) die Entwicklung einer Theorie sozialer Praktiken des Unterrichts, die ihren Ausgangspunkt, ,in der Analyse alltäglichen Unterrichts“ hat und „,auf das interaktive Geschehen im Rahmen der Unterrichtssituation“ fokussiert. In der Analyse wird danach gefragt, wie die Unterrichtspraxis auf den Zweck von Unterricht bezogen ist, Lernen zu ermöglichen. Für die Analyse wird Unterricht konzeptuell als ,ein Bündel aufeinander bezogener, ineinander verschränkter sozialer 
Praktiken“ gefasst (Breidenstein, 2008, S. 207). Ein Unterschied zum hermeneutischen Forschungsprogramm von Gruschka wird darin gesehen, dass in der Analyse nicht Sinn und Bedeutungen entschlüsselt, sondern durch ethnographische Forschung teilnehmend beobachtend Praktiken entdeckt werden sollen. Ethnographische Analyse orientiert sich dabei nicht primär an traditionellen pädagogischen Kategorien wie Didaktik, Erziehung oder Bildung, sondern vielmehr an identifizierten Phänomenen des Unterrichts wie etwa der Bewertung und Zurechnung von Leistungen zu Personen, Peer-Kultur der Schüler oder Langeweile im Unterricht. Erschlossen wird so, wie Interaktion durch Formen der Organisation von Unterricht beeinflusst wird und wie Lernen als ein Übergang von Nicht-Wissen zu Wissen kommuniziert wird.

In beiden Forschungsprogrammen werden Struktur- und Prozessmerkmale des Handelns im Unterricht rekonstruiert. Beide Programme zielen darauf ab, komplexe Phänomene des Unterrichtens zu beschreiben, und schlagen Wege seiner systematischen Reflexion vor. Gerade wegen der engen Nachbarschaft entstehen auf methodologischer Ebene große Überschneidungen in der Forschung zur Lehrer-Schüler-Interaktion (Lüders, 2011, S. 651), andererseits aber auch jeweils spezifische Sichtstärken einzelner Programme. So liegt etwa im objektiv-hermeneutischen Forschungsprogramm von Gruschka (2005, S. 45-92) der Fokus in der starken methodischen Absicherung der Interpretation. Demgegenüber wird in ethnographischen Arbeiten die Konstruktion von Datenmaterial durch die Erhebung betont, was situative Flexibilität in der Feldforschung erlaubt, die in objektiv-hermeneutischen Projekten bislang nicht ausgeschöpft wird. Spezifische Sichtstärken der Forschungsprogramme lassen sich auch an erhobenen Vorwürfen ausmachen: Ethnographischen Studien wird vorgeworfen, sie würden Merkmale des Interaktionsgeschehens durch Interpretation verdoppeln und strukturelle Rahmenbedingungen der Interaktion vernachlässigen. Strukturalistischen Programmen wird vorgeworfen, dass „Unterricht von den Funktionen der Schule für das Gesellschaftssystem her" analysiert wird (Breidenstein, 2010, S. 871), anstatt Phänomene des Unterrichts als eigenständige Kultur in Klassen zu untersuchen. In beiden Fällen scheinen tendenziell orthodoxe Positionen einen komplementären Einsatz von konzeptuellen Denkfiguren und methodischem Vorgehen in Erhebung und Analyse zu verhindern. Eine kohärenzerhaltende Variation von Elementen der Forschungsprogramme wäre eine Möglichkeit, um Komplementarität von Forschungsprogrammen zu Handeln im Unterricht auszuloten, Sichtstärken entlang spezifischer Forschungsinteressen weiterzuentwickeln und so der gemeinsamen Programmatik einer Theorie des Unterrichts nachzukommen.

\section{Literatur}

Alheit, P., \& Dausien, B. (2009). Bildungsprozesse über die Lebensspanne: Zur Politik und Theorie lebenslangen Lernens. In R. Tippelt \& B. Schmidt (Hrsg.), Handbuch Bildungsforschung (S. 713-734). Wiesbaden: VS Verlag für Sozialwissenschaften.

Arnold, K.-H. (2009). Lehr-Lern-Forschung ohne Allgemeine Didaktik? Über die Notwendigkeit einer integrierten Wissenschaft vom Unterricht. In K.-H. Arnold, S. Blömeke, R. Messner \& J. Schlömerkemper (Hrsg.), Allgemeine Didaktik und Lehr-Lernforschung. Kontrover- 
sen und Entwicklungsperspektiven einer Wissenschaft vom Unterricht (S. 13-25). Bad Heilbrunn: Klinkhardt.

Baumert, J., \& Kunter, M. (2006). Stichwort: Professionelle Kompetenz von Lehrkräften. Zeitschrift für Erziehungswissenschaft, 9(4), 469-520.

Blankertz, H. (1975). Theorien und Modelle der Didaktik (9., überarb. Aufl.). München: Juventa.

Blömeke, S. (2009). Allgemeine Didaktik ohne empirische Lernforschung? Perspektiven einer reflexiven Bildungsforschung. In K.-H. Arnold, S. Blömeke, R. Messner \& J. Schlömerkemper (Hrsg.), Allgemeine Didaktik und Lehr-Lernforschung. Kontroversen und Entwicklungsperspektiven einer Wissenschaft vom Unterricht (S. 13-25). Bad Heilbrunn: Klinkhardt.

Blömeke, S., \& Müller, C. (2008). Zum Zusammenhang von Allgemeiner Didaktik und LehrLernforschung im Unterrichtsgeschehen. In M. A. Meyer, M. Prenzel \& S. Hellekamps (Hrsg.), Perspektiven der Didaktik (Sonderheft der Zeitschrift für Erziehungswissenschaft 9/2008, S. 239-258). Wiesbaden: VS Verlag für Sozialwissenschaften.

Breidenstein, G. (2008). Allgemeine Didaktik und praxeologische Unterrichtsforschung. In M. A. Meyer, M. Prenzel \& S. Hellekamps (Hrsg.), Perspektiven der Didaktik (Sonderheft der Zeitschrift für Erziehungswissenschaft 9/2008, S. 201-218). Wiesbaden: VS Verlag für Sozialwissenschaften.

Breidenstein, G. (2010). Überlegungen zu einer Theorie des Unterrichts. Zeitschrift für Pädagogik, 56(6), 869-887.

Dausien, B. (2007). Reflexivität, Vertrauen, Professionalität. Was Studierende in einer gemeinsamen Praxis qualitativer Forschung lernen können. Forum Qualitative Sozialforschung, 8(1). http://nbn-resolving.de/urn:nbn:de:0114-fqs0701D4Da3 [20.01.2012].

de Jong, T. (2010). Cognitive load theory, educational research, and instructional design: some food for thought. Instructional Science, 38(2), 105-134.

Gage, N. L. (2009). A Conception of Teaching. New York: Springer.

Giesinger, J. (2006). Erziehung der Gehirne? Willensfreiheit, Hirnforschung und Pädagogik. Zeitschrift für Erziehungswissenschaft, 9(1), 97-109.

Gruschka, A. (2002). Didaktik. Das Kreuz mit der Vermittlung. Elf Einsprüche gegen den didaktischen Betrieb. Wetzlar: Büchse der Pandora.

Gruschka, A. (2005). Auf dem Weg zu einer Theorie des Unterrichtens. Die widersprüchliche Einheit von Erziehung, Didaktik und Bildung in der allgemeinbildenden Schule. Vorstudie (Frankfurter Beiträge zur Erziehungswissenschaft, Forschungsberichte, Bd. 5). Frankfurt a.M.: Fachbereich Erziehungswissenschaften der Johann Wolfgang Goethe-Universität.

Gruschka, A. (2009). Erkenntnis in und durch Unterricht. Empirische Studien zur Bedeutung der Erkenntnis- und Wissenschaftstheorie für die Didaktik. Wetzlar: Büchse der Pandora.

Gruschka, A. (2010). Verstehen lehren. Ein Plädoyer für guten Unterricht. Stuttgart: Reclam.

Gruschka, A. (2011). Pädagogische Forschung als Erforschung der Pädagogik. Eine Grundlegung. Opladen: Budrich.

Helmke, A. (2011). Forschung zur Lernwirksamkeit des Lehrerhandelns. In E. Terhart, H. Bennewitz \& M. Rothland (Hrsg.), Handbuch der Forschung zum Lehrerberuf (S. 630-643). Münster: Waxmann.

Helsper, W. (2002). Wissen, Können, Nicht-Wissen-Können. Wissensformen des Lehrers und Konsequenzen für die Lehrerbildung. In Zentrum für Schulforschung und Fragen der Lehrerbildung (Hrsg.), Die Lehrerbildung der Zukunft. Eine Streitschrift (S. 67-86). Opladen: Leske \& Budrich.

Helsper, W. (2011). Lehrerprofessionalität - der strukturtheoretische Professionsansatz zum Lehrberuf. In E. Terhart, H. Bennewitz \& M. Rothland (Hrsg.), Handbuch der Forschung zum Lehrerberuf (S. 149-170). Münster: Waxmann.

Herber, H.-J. (1997). Ein Paradigmenvergleich. „Interesse“ aus pädagogischer und psychologischer Sicht. Salzburger Beiträge zur Erziehungswissenschaft, 1(1), 41-49. 
Hericks, U. (2008). Bildungsgangforschung und die Professionalisierung des Lehrerberufs - Perspektiven für die Allgemeine Didaktik. In M. A. Meyer, M. Prenzel \& S. Hellekamps (Hrsg.), Perspektiven der Didaktik (Sonderheft der Zeitschrift für Erziehungswissenschaft 9/2008, S. 61-75). Wiesbaden: VS Verlag für Sozialwissenschaften.

Herzog, W., \& Markova, E. (2011). Anforderungen an und Leitbilder für den Lehrberuf. In E. Terhart, H. Bennewitz \& M. Rothland (Hrsg.), Handbuch der Forschung zum Lehrerberuf (S. 63-78). Münster: Waxmann.

Jörg, T., Davis, B., \& Nickmans, G. (2007). Towards a new, complexity science of learning and education. Educational Research Review, 2(2), 145-156.

Kaiser, A., Koch-Priewe, B., \& Stübig, F. (2007). Die kritisch-konstruktive Didaktik und aktuelle Kontroversen um die Allgemeine Didaktik. In B. Koch-Priewe, F. Stübig \& K.-H. Arnold (Hrsg.), Das Potential der Allgemeinen Didaktik. Stellungnahmen aus der Perspektive der Bildungstheorie von Wolfgang Klafki (S. 7-26). Weinheim/Basel: Beltz.

Klieme, E. (2006). Empirische Unterrichtsforschung. Aktuelle Entwicklungen, theoretische Grundlagen und fachspezifische Befunde. Zeitschrift für Pädagogik, 52(6), 765-773.

Klieme, E., \& Rakoczy, K. (2008). Empirische Unterrichtsforschung und Fachdidaktik. Zeitschrift für Pädagogik, 54(2), 222-237.

Lüders, M. (2011). Forschung zur Lehrer-Schüler-Interaktion/Unterrichtskommunikation. In E. Terhart, H. Bennewitz \& M. Rothland (Hrsg.), Handbuch der Forschung zum Lehrerberuf (S. 644-666). Münster: Waxmann.

Lüders, M., \& Rauin, U. (2008). Unterrichts- und Lehr-Lern-Forschung. In W. Helsper \& J. Böhme (Hrsg.), Handbuch der Schulforschung (S. 717-745). Wiesbaden: VS Verlag für Sozialwissenschaften.

Luhmann, N., \& Schorr, K.-E. (1982). Zwischen Technologie und Selbstreferenz. Fragen an die Pädagogik. Frankfurt a.M.: Suhrkamp.

Messner, R. (2007). Allgemeine Didaktik und Lehr-Lernforschung. Über die Ambivalenz ihrer Beziehung am Beispiel der Erforschung selbstständigen Lernens. In B. Koch-Priewe, F. Stübig \& K.-H. Arnold (Hrsg.), Das Potential der Allgemeinen Didaktik. Stellungnahmen aus der Perspektive der Bildungstheorie von Wolfgang Klafki (S. 43-60). Weinheim/Basel: Beltz.

Meyer, M. A., Prenzel, M., \& Hellekamps, S. (2008). Editorial: Perspektiven der Didaktik. In Dies. (Hrsg.), Perspektiven der Didaktik (Sonderheft der Zeitschrift für Erziehungswissenschaft 9/2008, S. 7-10). Wiesbaden: VS Verlag für Sozialwissenschaften.

Neuweg, G. H. (2011). Das Wissen der Wissensvermittler. In E. Terhart, H. Bennewitz \& M. Rothland (Hrsg.), Handbuch der Forschung zum Lehrerberuf (S. 451-477). Münster: Waxmann.

Oevermann, U. (1996). Theoretische Skizze einer revidierten Theorie professionalisierten Handelns. In A. Combe \& W. Helsper (Hrsg.), Pädagogische Professionalität. Untersuchungen zum Typus pädagogischen Handelns (S. 70-183). Frankfurt a.M.: Suhrkamp.

Paas, F. (1992). Training Strategies for Attaining Transfer of Problem-Solving Skill in Statistics: A Cognitive-Load Approach. Journal of Educational Psychology, 84(4), 429-434.

Reusser, K. (2006). Konstruktivismus - vom epistemologischen Leitbegriff zur Erneuerung der didaktischen Kultur. In M. Baer, M. Fuchs, P. Füglister, K. Reusser \& H. Wyss (Hrsg.), Didaktik auf psychologischer Grundlage: Von Hans Aeblis kognitionspsychologischer Didaktik zur modernen Lehr- und Lernforschung (S. 151-168). Bern: Hep.

Reusser, K. (2008). Empirisch fundierte Didaktik - didaktisch fundierte Unterrichtsforschung. Eine Perspektive zur Neuorientierung der Allgemeinen Didaktik. In M. A. Meyer, M. Prenzel \& S. Hellekamps (Hrsg.), Perspektiven der Didaktik (Sonderheft der Zeitschrift für Erziehungswissenschaft 9/2008, S. 219-237). Wiesbaden: VS Verlag für Sozialwissenschaften.

Rothkopf, E. (2008). Reflections on the Field: Aspirations of Learning Science and the Practical Logic of Instructional Enterprises. Educational Psychology Review, 20(3), 351-368. 
Schurz, G. (1993). Koexistenzweisen rivalisierender Paradigmen. Eine begriffsklärende und problemtypologisierende Studie (IPS Vorveröffentlichungsreihe, Bd. 2). Salzburg: Institut für Philosophie der Universität Salzburg.

Schurz, G. (2006). Einführung in die Wissenschaftstheorie. Darmstadt: Wissenschaftliche Buchgesellschaft.

Seidel, T. (2011). Lehrerhandeln im Unterricht. In E. Terhart, H. Bennewitz \& M. Rothland (Hrsg.), Handbuch der Forschung zum Lehrerberuf (S. 605-629). Münster: Waxmann.

Staub, F. (2006). Allgemeine Didaktik und Lernpsychologie. Zur Dynamisierung eines schwierigen Verhältnisses. In M. Baer, M. Fuchs, P. Füglister, K. Reusser \& H. Wyss (Hrsg.), Didaktik auf psychologischer Grundlage: Von Hans Aeblis kognitionspsychologischer Didaktik zur modernen Lehr- und Lernforschung (S. 169-179). Bern: Hep.

Terhart, E. (1999). Konstruktivismus und Unterricht. Gibt es einen neuen Ansatz in der Allgemeinen Didaktik? Zeitschrift für Pädagogik, 45(5), 629-647.

Terhart, E. (2002). Fremde Schwestern. Zum Verhältnis von Allgemeiner Didaktik und empirischer Lehr-Lern-Forschung. Zeitschrift für Pädagogische Psychologie, 16(2), 77-86.

Terhart, E. (2005). Über Traditionen und Innovationen oder: Wie geht es weiter mit der Allgemeinen Didaktik? Zeitschrift für Pädagogik, 51(1), 1-13.

Terhart, E. (2008). Allgemeine Didaktik: Traditionen, Neuanfänge, Herausforderungen. In M. A. Meyer, M. Prenzel \& S. Hellekamps (Hrsg.), Perspektiven der Didaktik (Sonderheft der Zeitschrift für Erziehungswissenschaft 9/2008, S. 13-34). Wiesbaden: VS Verlag für Sozialwissenschaften.

von Olberg, H.-J. (2004). Didaktik auf dem Wege zur Vermittlungswissenschaft? Eine Sammelbesprechung neuerer Veröffentlichungen. Zeitschrift für Pädagogik, 50(1), 119-131.

Wahl, D. (2001). Nachhaltige Wege vom Wissen zum Handeln. Beiträge zur Lehrerbildung, 19(2), 157-174.

Winkel, R. (2002). Die kritisch-kommunikative Didaktik. In H. Gudjons \& R. Winkel (Hrsg.), Didaktische Theorien (S. 93-112). Hamburg: Bergmann \& Helbig.

\begin{abstract}
Concepts of teaching - in the form of models, theories, or schemes - have been developed both in general didactics and in research on teaching and learning. Through an analysis of concepts of both paradigms, commonalities and differences in the conceptualizations of action will be identified. The analysis is carried out by breaking down into their components theories of both paradigms. On the basis of identified challenges of the conceptualization of action, conceptual, methodological, and empirical perspectives, through which action in classroom teaching can be examined, will be sketched in the contribution's résumé.
\end{abstract}

Keywords: Didactics, Teaching-Learning-Research, Action, Conceptualisation, Teaching Research

\author{
Anschrift des Autors \\ Mag. Clemens Wieser, Institut für Bildungswissenschaft, Sensengasse 3a, \\ 1010 Wien, Österreich \\ E-Mail: clemens.wieser@univie.ac.at
}

